\title{
Secretory Carcinoma of the Breast: An Elusive Presentation of This Rare Pathology
}

\author{
Joseph Brandt, Jack Morgani, Alan Heimann and Edna Kapenhas*
}

The Ellen Hermanson Breast Center, Stony Brook Southampton Hospital, Southampton, USA

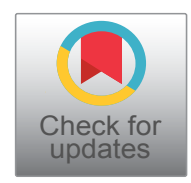

*Corresponding author: Edna Kapenhas, MD, The Ellen Hermanson Breast Center, Stony Brook Southampton Hospital, Southampton, NY 11968, USA, E-mail: EKapenhas@southamptonhospital.org

\begin{abstract}
Since first being noted in the literature in 1966, Secretory Breast Carcinoma (SBC) continues to be better defined in terms of its clinical, histologic, and genetic features. However, its unique presentation can elude seasoned practitioners from making a clinical diagnosis. Furthermore, there is neither consensus nor guidelines as to the optimal treatment approach for SBC. We are presenting this case report and literature review to raise awareness about SBC by demonstrating how uniquely this pathology can present itself, to summarize the current literature, and to present our specific approach to this rare cancer.
\end{abstract}

\section{Keywords}

Breast cancer, Secretory breast carcinoma, Triple negative, Entrectinib, ETV6-NTRK3

\section{Introduction}

First described as "juvenile carcinoma" by McDivitt and Stewart in 1966, [1] this unique pathology has since been defined as Secretory Breast Carcinoma (SBC) owing to the copious intra- and extracellular secretory material seen on histologic analysis. Secretory breast carcinoma is described in both men and women ranging from 3 to 86-years-old; clearly afflicting more than the juvenile population [2-4]. Due to its low incidence (0.015\% of all breast cancers) [3] and unique pathologic features, the diagnosis of SBC can be initially difficult to make.

Clinically, SBC typically presents early as a palpable mass. It is most often receptor triple negative (ER, PR, and HER2 negative) but low grade. Histologically, SBC presents as a solid, tubular, microcystic and rarely papillary pattern [5]. Due to the presence of secretory ma- terial, samples are strongly S-100 protein reactive. Secretory breast carcinoma is driven by the fusion gene ETV6-NTRK3 that results from a balanced translocation $\mathrm{t}(12 ; 15)[6]$.

Fortunately for most diagnosed with SBC, the course is indolent even in the setting of nodal metastasis, which is present in $15-35 \%$ cases at presentation $[2,3,7]$. Although distant metastasis is very rare, local recurrence is described, recurring as far as 16 years after treatment [8]. At present the primary treatment remains surgical.

\section{Case Report}

A 75-year-old female presented to our clinic with complaint of new onset right nipple pain and swelling for 4 weeks. Family history was significant for breast cancer in a maternal and a paternal first cousin, as well as a maternal aunt who succumbed to her disease at age 50 . The initial physical exam revealed a tender, erythematous, enlarged right nipple, but no palpable masses. The initial impression was mastitis, and she was started on azithromycin. A diagnostic mammogram showed asymmetry of the right nipple compared to the left (Figure 1), however, ultrasound at that time was unremarkable. Her symptoms did not improve with antibiotics.

A repeat targeted ultrasound of this area revealed an abnormal right nipple complex (Figure 2). She was then assessed by a breast surgeon, and the exam at that time revealed a firm, tender, erythematous mass of the superior aspect of the right nipple, and a palpable right axillary lymph node. Ultrasound-guided fine needle aspiration biopsy of the axillary node was consistent with metastatic adenocarcinoma. The tumor stained positive

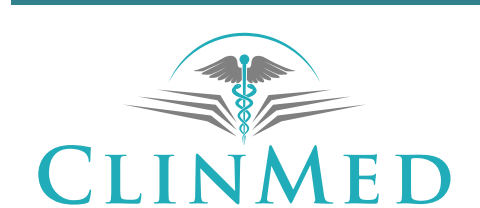

INTERNATIONAL LIBRARY

Citation: Brandt J, Morgani J, Heimann A, Kapenhas E (2017) Secretory Carcinoma of the Breast: An Elusive Presentation of This Rare Pathology. Int J Oncol Res 1:002.

Received: September 21, 2017; Accepted: February 24, 2018; Published: February 26, 2018

Copyright: (c) 2017 Brandt J, et al. This is an open-access article distributed under the terms of the Creative Commons Attribution License, which permits unrestricted use, distribution, and reproduction in any medium, provided the original author and source are credited. 

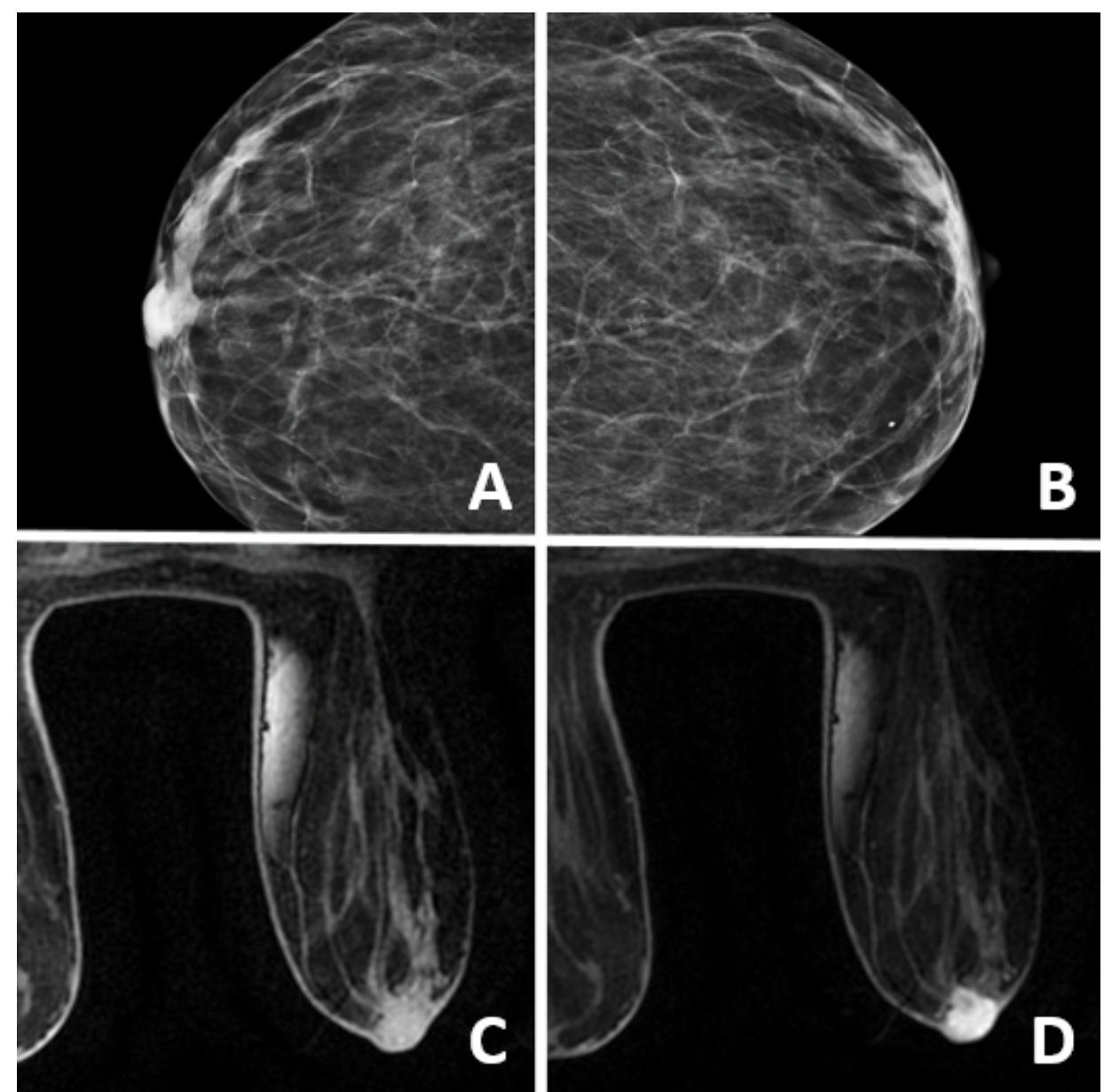

Figure 1: Radiographic findings $(A, B)$ Diagnostic bilateral mammogram with 3D tomosynthesis, CC projections demonstrate asymmetry of the right $(A)$ nipple when compared to the left $(B) ;(C, D)$ Breast MRI, T1 weighted Pre (C) and Post (D) contrast images showing $14 \times 14 \mathrm{~mm}$ abnormal enhancement of the right nipple.

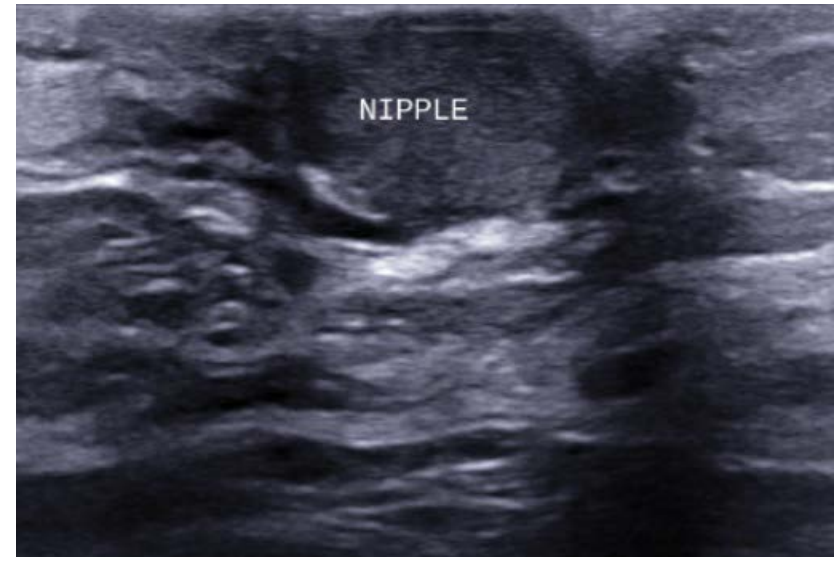

Figure 2: Targeted ultrasound of the right breast demonstrating retracted nipple, enlarged and asymmetric when compared to left.

for GATA3 which is consistent with a breast primary but could also be present in a primary skin cancer. A breast MRI (Figure 1) revealed a $14 \times 14 \mathrm{~mm}$ enhancement involving the right nipple. A subsequent punch biopsy of the right nipple mass revealed a triple negative adenocarcinoma. Morphologically, the skin biopsy tissue did not appear to be a primary skin tumor.

Given these findings, a primary breast cancer was suspected and she underwent a right central partial mastecto- my with axillary lymph node dissection. Pathology revealed a $1.3 \mathrm{~cm}$ invasive carcinoma, as well as DCIS with 1 of 21 lymph nodes positive for metastatic carcinoma. The tumor was positive for S-100, triple negative (ER, PR, and Her2), with a Ki-67 of $15 \%$. Molecular testing showed positivity for ETV6 and NTRK3 on Fluorescence In-Situ Hybridization (FISH). These morphologic, immunohistochemical and molecular findings were diagnostic of secretory carcinoma of the breast (Figure 3).

Adjuvant chemotherapy was not recommended as SBC rarely metastasizes to distant locations, and there is a paucity of data to show any benefit of systemic therapy. A consultation with the radiation oncologist was recommended, but the patient declined. Our plan is to follow her with clinical exams every 3-4 months, tumor markers, PET/CT every six months for two years, followed by once annually for three additional years. At present, the patient remains disease free at eight months after surgery.

\section{Discussion}

Overall, secretory breast carcinoma is a rarely encountered clinical entity accounting for less than $0.015 \%$ of all breast cancers [3]. The reported female to male ratio is $6: 1,[1,9]$ and SBC has been reported in almost every age group. As was demonstrated by this case, the 


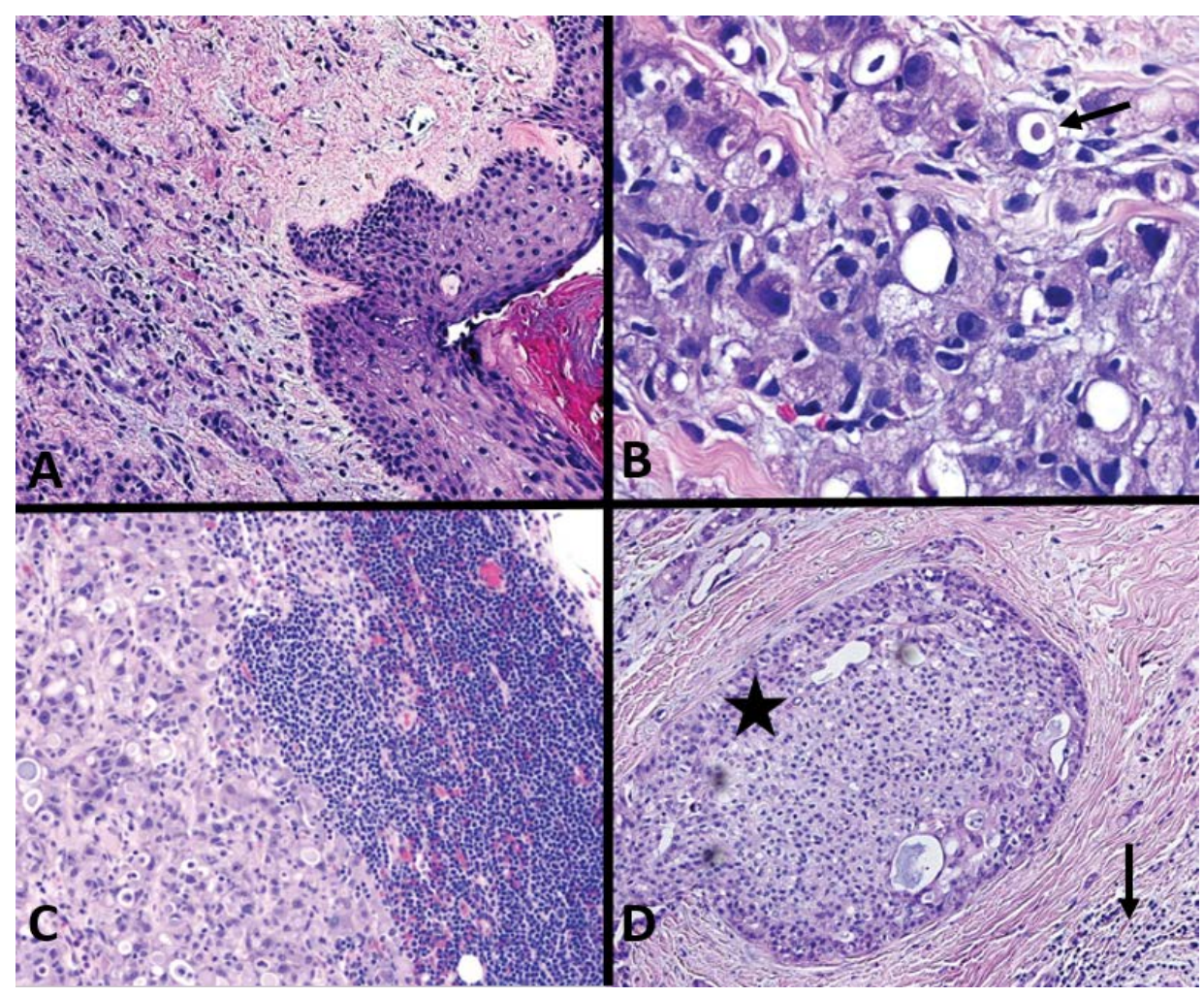

Figure 3: (A) Normal skin surface with underlying dermal tumor, 100x; (B) Dermis with tumor cells, with frothy granular, relatively abundant cytoplasm and intracytoplasmic vacuoles containing secretory material (arrow), nuclei have conspicuous nucleoli, 400x; (C) Lymph node metastasis, 100x; (D) Intraductal cancer (star) surrounded by invasive tumor (arrow), 100x.

diagnosis can initially be elusive. After failing treatment for presumed mastitis, a more thorough physical exam revealed a small nipple mass. This is consistent with existing data that shows that SBC most often presents as a palpable mass, [1] and although it can affect any quadrant and even the axilla, it often involves the nipple areolar complex, especially in males [4]. At the time of presentation, $15-35 \%$ of patients have been noted to have nodal metastasis. Despite this, distant metastasis remains exceedingly rare, and our literature review only was able to uncover five reported cases [7].

Pathologically, SBC is typically triple negative but low-grade, and the copious amount of intra/extracellular secretions make the tumors strongly S-100 reactive [5]. Secretory breast carcinoma has also recently been noted to belong to the basal-like spectrum of breast carcinomas. This was noted by Lae, et al. who found that in addition to being triple negative, SBC expressed CK5/6 and/or CK14 and/or KIT [10]. In our case, pathologic analysis was not able to make a definitive diagnosis until the specimen was sent for expert consultation and genetic testing. FISH revealed the presence of an ETV6NTRK3 fusion gene, which in the setting of breast cancer is pathomnemonic for SBC. This gene is a result of a balanced translocation $\mathrm{t}(12 ; 15)$, and was known to be associated with congenital fibrosarcoma and mesoblactic nephroma. However, it was not studied in relation to breast cancer until 2002. Although not present in every case of SBC, it is present in the vast majority of cases and is not seen in any other forms of breast cancer [6]. Ex- pression of this ETV6-NTRK3 gene leads to formation of a chimeric tyrosine kinase that activates the Ras-MAPK and inositol-3'-kinase Akt pathways, which are noted to be the first event in the oncogenesis of SBC [11,12]. Furthermore, it was shown that a retroviral transfer of the same fusion gene induced SBC in mice [6].

The existing data supports surgery as the primary treatment. Partial mastectomy is a reasonable approach to most tumors, with total mastectomy reserved for cancers not amenable to breast conserving surgery (cosmetic result, multi-centric disease, patient desire, etc.). Regarding nodal sampling, there is no consensus guideline at present, but a recent review of data from Survival, Epidemiology, and End Results (SEER) database showed that as many as $34.9 \%$ of patients can have positive nodes at the time of presentation. As a result Horowitz, et al. have recommended nodal sampling with a minimum of a sentinel lymph node biopsy be performed [3]. The same study also examined the role of radiation therapy in regards to SBC. Ten year cause-specific survival was found to be $97.1 \%$ in patients treated with surgery (partial mastectomy or mastectomy) and subsequent radiation therapy versus $89.2 \%$ in the cohort treated with surgery alone, but this data was not statistically significant. Regarding chemotherapy, there remains paucity of data to support systemic treatment, but with metastatic disease being so rare, it is difficult to justify its use. In terms of hormonal blockade, most $\mathrm{SBC}$ is not hormone responsive. In the rare case of a hormone receptor positive SBC, hormonal blockade would 
be reasonable, but again there is no specific data to support its use in SBC. Recently, a novel tyrosine kinase inhibitor that targets NTRK3, Entrectinib, was developed and is currently in phase II clinical trials (STARTRK-2) with promising preliminary results [13].

Our patient did not desire any further treatment beyond the central partial mastectomy with axillary node dissection. Given the multiple reports of reasonably long disease-free intervals (even in the face of positive lymph nodes), and limited data on the benefit of chemo or radiation therapy, a plan for close clinical follow-up was ultimately chosen. In the event that our patient has a local recurrence, further resection will be the primary treatment. Additionally, if local recurrence or evidence of distant metastasis is noted in this patient, we will strongly recommend enrollment in the STARTRK-2 trial as initial data shows that Entrectinib has reasonable efficacy and is well tolerated [13].

\section{Conclusion}

Overall, secretory breast carcinoma should be treated in a similar fashion to all breast cancers. Understanding patient goals of care and utilizing a multi-disciplinary approach to patient care ultimately yields the best results while minimizing morbidity. We believe that local resection with partial mastectomy or total mastectomy remains the corner stone of treatment. Nodal sampling with either sentinel lymph node biopsy or formal axillary dissection should be obtained due to the reported frequency of nodal metastasis. Beyond that, the decision to pursue chemotherapy, radiation, hormonal therapy, or other novel treatments is highly individualized.

\section{Acknowledgment}

Authors report no conflict of interest or financial disclosures.

\section{References}

1. McDivitt RW, Stewart FW (1966) Breast carcinoma in children. JAMA 195: 388-390.

2. Dali Li, Xiuying Xiao, Wentao Yang, Ruohong Shui, Xiaoyu Tu, et al. (2012) Secretory breast carcinoma: A clinicopathological and immunophenotypic study of 15 cases with a review of the literature. Modern Pathology 25: 567-575.
3. David P Horowitz, Charu S Sharma, Eileen Connolly, Daniela Gidea-Addeo, Israel Deutsch (2012) Secretory Carcinoma of the Breast: Results from the survivial, epidemiology and end result database. Breast 21: 350-353.

4. Jinhua Ding, Li Jiang, Yongli Gan, Weizhu Wu (2015) A rare case of secretory breast carcinoma in a male adult with axillary lymph node metastasis. Int $\mathrm{J}$ Clin Exp Pathol 8: 3322-3327.

5. Ruohong Shui, Yufan Cheng, Qianming Bai, Wentao Yang (2017) Secretory breast carcinoma with a papillary predominant pattern-an uncommon morphology variant. Histopathology 71: 488-493.

6. Tognon C, Knezevich SR, Huntsman D, Roskelley CD, Melnyk N, et al. (2002) Expression of the ETV6-NTRK3 gene fusion as a primary event in human secretory breast carcinoma. Cancer Cell 2: 367-376.

7. Arce C, Cortes-Padilla D, Huntsman DG, Miller MA, Dueñnas-Gonzalez A, et al. (2005) Secretory carcinoma of the breast containing the ETV6-NTRK3 fusion gene in a male; case report and review of literature. World J Surg Oncol 3: 35.

8. Herve Tixier, Anne Picard, Severine Guiu, Coudert B, Loustalot C, et al. (2011) Long-term reccurance of secretory carcinoma with metastatic sentinel lymph nodes. Arch Gynecol Obstet 283: 77-78.

9. Serour F, Gilad A, Koplovic J, Krispin M (1992) Secretory breast cancer in childhood and adolescence: Report of a case and review of the literature. Med Pediatr Oncol 20: 341-344.

10. Marick Lae, Paul Freneaux, Xavier Sastre-Garau, Chouchane O, Sigal-Zafrani B, et al. (2009) Secretory breast carcinomas with ETV6-NTRK3 fusion gene belong to the basal-like carcinoma spectrum. Mod Pathol 22: 291-298.

11. Marie Del Castillo, Frederic Chibon, Laurent Arnould, Croce S, Ribeiro A, et al. (2015) Secretory Breast Carcinoma: A histopatologic and genomic spectrum characterized by a joint specific ETV6-NTRK3 gene fusion. Am J Surg Pathol 39: 1458-1467.

12. Elena Ardini, Maria Menichincheri, Patrizia Banfi, Bosotti $R$, De Ponti C, et al. (2016) Entrectinib, a Pan-TRK, ROS1 and ALK inhibitor with Activity in Multiple Molecularly Defined Cancer Indications. Mol Cancer Ther 15: 628-639.

13. Alexander Drilon, Salvatore Siena, Sai-Hong Ignatius Ou, $\mathrm{Pa}-$ tel M, Ahn MJ, et al. (2017) Safety and Antitumor Activity of the Multitargeted Pan-TRK, ROS1, and ALK Inhibitor Entrectinib: Combined Results from Two Phase I Trials (ALKA-372-001 and STARTRK-1). Cancer Discov 7: 400-409. 